\title{
Perméabilité estimée par la granulométrie Proposition d'une méthode et test de son efficacité
}

V. SAVATIER

Fondasol agence

de Montpellier

Bât, A, bureaux d'Olympie 134, av, de Palavas 34070 Montpellier
Après une analyse des relations entre les formules de Kozeny et de Hazen, nous établirons, à partir de la formule de Kozeny, une nouvelle relation granulométrieperméabilité utilisable surs des sols de granulométrie étalée dont on ne connaît pas a priori la porosité. Nous montrons, sur un échantillon de 21 sols, que la relation proposée donne des résultats plus satisfaisants que la formule de Hazen utilisée lorsque $\frac{\mathrm{d}_{60}}{\mathrm{~d}_{10}}>2$ et que la probabilité d'avoir une estimation ne différant pas de plus d'un facteur 10 de la perméabilité vraie est de $80 \%$,

\section{Correlation between permeability and granulometry Proposition of a new method}

We propose a new method of estimation of permeability based on Konezy formula.

We show that this method is better than. Hazen formula employed when $\frac{d_{\text {fin }}}{d}>2$.

The probability to obtain with our method a factor 10 over or under estimation of permeability is less than $10 \%$.
} 
L'existence d'une relation entre la granulométrie d'un sol et sa perméabilité peut sembler une évidence. L'utilisation d'une telle relation pour déterminer la perméabilité d'un sol présente pourtant de nombreuses difficultés liées, d'une part, à l'hétérogénéité des sols et, d'autre part, au fait que la granulométrie n'exprime pas l'organisation des pores de l'aquifère, enfin aux difficultés de définition de la granulométrie pour des sols aux grains allongés.

Ces difficultés conduisent de nombreux professionnels à nier toute utilité aux approches de la perméabilité par la granulométrie et à préconiser, à juste titre, un recours systématique aux essais de pompage et parfois, à moins juste titre, un recours systématique aux essais d'eau ponctuels par injection de type Lefranc, Nasberg ou Porcher. D'autres professionnels insistant sur la possible faible fiabilité des essais d'eau par injection du fait des phénomènes de colmatage leur préfèrent, en l'absence de pompage d'essai, l'utilisation de la formule de Hazen qui est fréquemment utilisée sans tenir compte de son domaine de validité restreint aux sols isogranulaires à grains sphériques.

L'origine des erreurs à craindre lors des essais d'eau par injection étant différente de celle à prévoir par les estimations à partir des analyses granulométriques, il peut ètre tentant d'utiliser les deux approches. L'obtention de deux résultats similaires est, en effet, un gage de bonne qualité de l'estimation, et l'analyse de l'origine des différences peut permettre d'éviter les déboires auxquels l'utilisation injustifiée de l'une ou l'autre des méthodes aurait conduit.

Pour cela, il nous parait nécessaire de disposer d'une méthode d'estimation à partir de la granulométrie ayant un domaine d'application plus large que la formule de Hazen.

Le propos de cet article est de proposer une telle relation et d'examiner si elle constitue un progrès par rapport à l'utilisation de la formule de Hazen hors de son domaine d'application.

\section{1}

\section{Rappel des formules de Hazen et de Kozeny}

\section{1}

\section{La formule de Hazen}

Cette formule qui a, d'apres Schneebeli, une origine expérimentale est valable pour un sol isogranulaire. Son domaine de validité est en effet restreint aux sols tels que $\frac{\mathrm{d}_{60}}{\mathrm{~d}_{10}} \leq 2$ ( $\mathrm{d}_{n}$ est de diamètre des grains correspondant à un passant de $\mathrm{n} \%$ ).

Elle vaut

- perméabilité intrinsèque $: K=\frac{\left(\mathrm{d}_{10}\right)^{2}}{1000}$

- perméabilité à l'eau : $\mathrm{k}(\mathrm{m} / \mathrm{s})=\left(\mathrm{d}_{10}\right)^{2} \mathrm{~d}_{10}$ en $\mathrm{cm}$

Rappelons au passage que la perméabilité à l'eau se déduit de la perméabilité intrinsèque par la formule: $\mathrm{K}=\frac{\eta}{\gamma_{W}} \mathrm{k}$

avec: $\eta \quad$ viscosité dynamique de l'eau valant à $20^{\circ}: \eta=10^{-6}$ kPa.s ;

nw poids spécifique de l'eau : $m=10 \mathrm{kN} / \mathrm{m}^{3}$

On a done:

$$
\left[\mathrm{K}\left(\mathrm{m}^{2}\right)=10^{-7} \mathrm{k}(\mathrm{m} / \mathrm{s})\right]
$$

En toute rigueur, la formule de Hazen ne devrait pas être utilisée hors de son domaine de validité.

\section{2}

\section{La formule de Kozeny Fair et Hatch}

Ainsi que cela est montré par Schneebeli [1] et Guéguen et Palciauskas [2], cette formule a des bases théoriques. Elle correspond à une modélisation de l'aquifère par un réseau de tubes. Nous avons perméabilité intrinséque : $K=\frac{a}{S_{0}^{2}} \frac{n^{3}}{(1-n)^{2}}$

a coefficient de proportionnalité proche de 1;

n porosité ;

S surface des pores par unitê de volume de solide (sans pore).

Remarquons que dans un sol granulaire, on a $S_{0}=\frac{S}{V}$ avec :

$S$ surface des grains ;

$\mathrm{V}$ volume des grains.

Cette formule peut donc s'écrire sous la forme suivante donnée par Schneebeli dans la référence [1]

$$
\text { perméabilité intrinsèque: } K=a\left(\frac{V}{S}\right)^{2} \frac{n^{3}}{(1-n)^{2}}
$$

Du fait de son mode d'établissement, cette formule doit être réservée aux sols granulaires où la surface du grain correspond à la surface des pores.

L'utilisation de cette formule est difficile, car elle nécessite de connaitre la porosité, le volume des grains ainsi que leur surface par unité de volume.

Elle peut, par contre, ètre utilisée pour des sols granulaires à granulométrie étalée sous réserve d'une répartition homogène des grains.

\section{2}

\section{Méthode de détermination de la perméabilité pour des sols de granulométrie étendue}

Nous repartirons de la formule de Kozeny en supposant, ce qui est le cas pour la plupart des sols alluviaux. que les grains peuvent étre remplacés par des sphères.

Soit un sol hétérogranulaire comportant i classes granulaires sphériques, on a :

- volume des grains : $\mathrm{V}=\sum_{i}$ niVi avec $\mathrm{Vi}=\frac{\pi(\mathrm{di})^{3}}{6}$

- surface des grains : $\mathrm{S}=\sum$ niSi avec $\mathrm{Si}=\pi(\mathrm{di})^{2}$.

Dans chaque classe granulaire, le nombre de grains dans un échantillon est : $\mathrm{ni}=\frac{\mathrm{x}_{\mathrm{P}} \mathrm{P}_{\mathrm{T}}}{\mathrm{Vi} \gamma_{\mathrm{S}}}$ 
$\mathrm{x}_{i} \quad$ pourcentage pondèral des grains de diamètre di

$\mathrm{P}_{\mathrm{T}}$ poids total de l'échantillon sec ;

Vi volume d'un grain de diamètre di ;

$\gamma_{s}$ poids spécifique des grains.

On a alors: $\mathrm{V}=\sum \frac{\mathrm{x}_{1} \mathrm{P}_{\mathrm{T}}}{\mathrm{V}_{i \gamma_{5}}} \cdot \mathrm{V}_{\mathrm{i}}=\frac{\mathrm{P}_{\mathrm{T}}}{\gamma_{5}} \sum \mathrm{x}_{i}$

$$
\mathrm{S}=\sum \frac{\mathrm{x}_{\mathrm{P}} \mathrm{P}_{\mathrm{T}}}{\mathrm{Vi} \gamma_{5}}, \mathrm{Si}=\frac{\mathrm{P}_{\mathrm{T}}}{\gamma_{5}} \sum \mathrm{x}_{\mathrm{I}} \frac{\mathrm{Si}}{\mathrm{Vi}}=\frac{\mathrm{P}_{\mathrm{T}}}{\gamma_{5}} \sum \frac{6}{\mathrm{di}} \mathrm{x}_{\mathrm{i}}
$$

On a donc: $\frac{V}{S}=\frac{\sum x_{i}}{6 \sum \frac{x_{i}}{d i}}=\frac{1}{6 \sum \frac{x_{i}}{d i}}$

La formule de Kozeny s'écrit donc pour un sol constitué de i classes granulaires

$$
K=\frac{a}{36}\left(\frac{1}{\sum \frac{x_{i}}{d i}}\right)^{2} \frac{n^{3}}{(1-n)^{2}}
$$

avec:

a coefficient proche de 1 ;

$x$ pourcentage pondéral des sols de diamètre di ;

n porosité.

On peut remarquer que dans le cas d'un sol isogranulaire cette formule donne:

$$
K=\frac{a}{36}\left(d_{10}\right)^{2} \frac{n^{3}}{(1-n)^{2}}
$$

On retrouve donc la formule de Hazen sous réserve que :

$$
\frac{a}{36} \frac{n^{3}}{(1-n)^{2}}=10^{-3}
$$

11 apparait donc que la formule de Hazen ne serait valable que pour une certaine porosité.

On doit cependant remarquer que dans les sables et les limons, l'indice des vides est compris entre 0,4 et 1. on a done:

$$
0,286<n<0,5 \text { d'où } 0,046<\frac{n^{3}}{(1-n)^{2}}<0,5
$$

La plage maximale de variation des indices des vides n'entraine donc qu'une incertitude d'un facteur 10 sur la perméabilité. La plage de variation des indices des vides de sol isogranulaire sédimentaire étant plus faible, cela explique que la formule de Hazen puisse être utilisée avec un certain succès

Si l'on s'intéresse à la perméabilité à l'eau du sol, on montre facilement à partir des formules (3) et (6) que:

$$
k(m / s)=\frac{a}{36} \cdot 10^{7}\left(\frac{1}{\sum \frac{x_{i}}{d i}}\right)^{2} \frac{n^{3}}{(1-n)^{2}}(\text { di en } m)
$$

soit : $\mathrm{k}(\mathrm{m} / \mathrm{s})=\frac{\mathrm{a}}{36} \cdot 10^{3}\left(\frac{1}{\sum \frac{x_{i}}{\mathrm{di}}}\right)^{2} \frac{\pi^{3}}{(1-\mathrm{n})^{2}}$ (di en $\left.\mathrm{cm}\right)$
Cette formule peut ètre utilisée pour calculer l'ordre de grandeur de la perméabilité lorsque l'on connait la porosité efficace.

Si l'on ne connait pas la porosité efficace, on peut écrire, en remarquant que la porosité des limons et des sables est $0,286<n<0,5$ et en considérant que la porosité efficace est, pour les sables et les limons, de l'ordre de $20 \%$ de la porosité totale :

$$
0,25 \mathrm{a}\left(\frac{1}{\sum \frac{\mathrm{x}}{\mathrm{di}}}\right)^{2}<\mathrm{k}(\mathrm{m} / \mathrm{s})<2,77 \mathrm{a}\left(\frac{1}{\sum \frac{\mathrm{x}_{i}}{\mathrm{di}}}\right)^{2} \text { (di en cm) }
$$

Ce que lion peut écrire

$$
k(m / s)=\alpha\left(\frac{1}{\sum \frac{x_{i}}{d i}}\right)^{2} \text { (di en cm) }
$$

avec $: 0,25<\alpha<2,8$

Si l'on sépare le sol en classe granulométriques correspondant à $20 \%$ de la masse totale de materjaux, la formule (8) s'écrit:

$$
k_{G}=a\left(\frac{5}{\frac{1}{d_{10}}+\frac{1}{d_{30}}+\frac{1}{d_{50}}+\frac{1}{d_{70}}+\frac{1}{d_{80}}}\right)^{2}
$$

avec : $0,25<\alpha<2,8$;

di diamètre en centimètre du grain correspondant à un refus de $1 \%$;

$k_{G}$. perméabilité estimée à partir de l'analyse granulométrique.

C'est cette formule que nous utiliserons dans la suite.

\section{3}

\section{Comparaison des perméabilités estimées par les formules précédentes avec des résultats d'essais de pompage}

De façon à tester l'utilité de la formule (9), nous avons regroupé, clans le tableau I, 21 courbes granulométriques correspondant à des sols dont la perméabilité avait été mesurée par des essais de pompage. Nous donnons dans ce tableau et pour chaque courbe granulométrique, outre la perméabilité mesurée par pompage, la perméabilité pouvant ètre estimée par la formule de Hazen et celle estimée par la formule (9) en retenant $\alpha=1$.

Étudions la corrélation existant entré ces différentes perméabilités ou plutôt entre leurs logarithmes népériens. 
TABLEAUI Comparaison des granulométries et des perméabilités de 21 sols.

\begin{tabular}{|c|c|c|c|c|c|c|c|c|c|c|}
\hline $\begin{array}{l}\text { Chantier } \\
\text { sondagefformation }\end{array}$ & Echantillon & DI0 सm & D30 cm & D50 cm & D70 cm & $\mathrm{D} 90 \mathrm{sm}$ & $\Sigma(t / D x)$ & $\begin{array}{l}\mathrm{k} \text {. Fiazen } \\
\mathrm{mi} / \mathrm{s}\end{array}$ & $\begin{array}{c}\text { kpompage } \\
\mathrm{m} / \mathrm{s}\end{array}$ & $\frac{k}{m} / s$ \\
\hline \multicolumn{11}{|l|}{ port Mariane } \\
\hline pait & $5-6 m$ & $8 E-04$ & 0.006 & 0.02 & 0.04 & 0.08 & 1504 & $6.40 E-07$ & $7.20 \mathrm{E}-06$ & 1.10E-05 \\
\hline$n 1$ & $5,5 \cdot 6.5 \mathrm{~m}$ & $8 E-04$ & 0,005 & 0,015 & 0,045 & 0.15 & 1568 & $6,40 E-07$ & $7,20 E-06$ & $1,02 \mathrm{E}=05$ \\
\hline$n 2$ & $4,3-5,3 \mathrm{~m}$ & 0,001 & 0,005 & 0,015 & 0,045 & 0,1 & 1321 & $1,00 E-06$ & $7,20 \mathrm{E}-06$ & $1.43 E-05$ \\
\hline n3 & $4,5 \cdot 5,5 \mathrm{~m}$ & $8 E-04$ & 0,003 & 0,007 & 0,02 & 0,08 & 1855 & $6,40 E-07$ & $7,20 E-06$ & $7,26 \mathrm{E}-26$ \\
\hline$n 4$ & $4,3-5,3$ แล & eE-04 & 0,002 & 0,004 & 0,009 & 0,07 & 2292 & $6.40 E-07$ & $7,20 E-06$ & $4.76 \mathrm{E}-06$ \\
\hline n5 & $3.7-4.7 \mathrm{~m}$ & $8 E-04$ & 0.003 & 0.006 & 0.05 & 0.3 & 1773 & $6,40 E-07$ & $7,20 E-06$ & 7,95E-06 \\
\hline$n 6$ & $4-5 \mathrm{~m}$ & $8 E-04$ & 0,002 & 0,006 & 0,03 & 0,3 & 1968 & $6.40 \mathrm{E}-07$ & $7,20 \mathrm{E}-06$ & $6.45 E-06$ \\
\hline 14 & $8,5-10 \mathrm{~m}$ & 0,003 & 0,07 & 0.7 & 2.5 & 6 & 350 & $9,00 E-06$ & $1,00 \mathrm{E}-03$ & 2.05E-04 \\
\hline 54 & $10-11,5$ hatit & 0,012 & 0,12 & 5 & 8 & 15 & 92 & $1,44 \mathrm{E}-04$ & $1,00 E-03$ & 2,95E-03 \\
\hline f4 & $10-11.5$ bas & 0,002 & 0,03 & 0,055 & 0.08 & 0,15 & 571 & $4,00 E-06$ & $1,00 E-03$ & $7,58 E-0.5$ \\
\hline \multirow[t]{2}{*}{ sable de Fontainebleau } & fin & $2 E-04$ & 0,0016 & 0,01 & 0,012 & 0,015 & 5417 & $4,00 E-08$ & $1,00 E-06$ & $8.52 E-07$ \\
\hline & grossier & 0.012 & 0,019 & 0,02 & 0,02 & 0,04 & 261 & $1,44 E-04$ & $1,00 \mathrm{E}-04$ & $3,67 \mathrm{E}-04$ \\
\hline $\begin{array}{l}\text { sable de Beauchamps: } \\
\text { en tète de couche }\end{array}$ & $\min$ & $\begin{array}{l}0,015 \\
0.02\end{array}$ & $\begin{array}{l}0,023 \\
0,038\end{array}$ & $\begin{array}{c}0,028 \\
0,05\end{array}$ & $\begin{array}{l}0,035 \\
0.0699\end{array}$ & $\begin{array}{l}0,062 \\
0,125 \\
\end{array}$ & $\begin{array}{l}191 \\
119 \\
\end{array}$ & $\begin{array}{l}2,25 E-04 \\
4,00 E-04\end{array}$ & $\begin{array}{l}1,00 \mathrm{E}-05 \\
1,00 \mathrm{E}-04\end{array}$ & $\begin{array}{l}6,88 \mathrm{E}-04 \\
1,77 \mathrm{E}-03\end{array}$ \\
\hline \multirow[t]{2}{*}{ sable de Cuise } & min & $2 E-04$ & $2 E=04$ & $9 E-04$ & 0.0003 & 0,007 & 11587 & $4,00 E-08$ & $1,00 \mathrm{E}-06$ & $1,86 \mathrm{E}-07$ \\
\hline & $\max$ & 0,002 & 0,06 & 0,08 & 0,18 & 0,4 & 537 & $4,00 \mathrm{E}-06$ & $1,00 E-04$ & $8,66 \mathrm{E}-0,5$ \\
\hline \multicolumn{11}{|l|}{ falluvions du Doubs } \\
\hline $5 \operatorname{ci032-1}$ & $4,8-6,1$ & 0,02 & 0,3 & 0.8 & 1.8 & 3,8 & 55 & $4,00 E-04$ & $6.40 E-03$ & $8.14 \mathrm{E}-03$ \\
\hline $5 c 3032-2$ & $3,6-5,5$ & 0,06 & 0,31 & 0.7 & 1.3 & 2.8 & 22 & $3,60 \mathrm{E}-03$ & $7,30 E-03$ & $4,96 E-02$ \\
\hline \multicolumn{11}{|l|}{ argile sableuse } \\
\hline $5 \operatorname{cs} 3032-1$ & $8,7-10$ & $1 E-04$ & $2 E \cdot 04$ & $5 E-04$ & 0,004 & 0,009 & 19028 & $1,00 \mathrm{E}-08$ & $5,00 \mathrm{E}-07$ & $6,91 \mathrm{E}-08$ \\
\hline $503032-2$ & $8.5-9.5$ & $1 E-04$ & $0,00 t$ & 0,005 & 0,013 & 1.2 & 11111 & $1.00 E-08$ & $8,00 E-06$ & $2,03 E-07$ \\
\hline $\begin{array}{l}\text { Nimes Sistre graveleux } \\
\text { sc2 }\end{array}$ & $3,4-4,9$ & 0,008 & 0,05 & 0,07 & 0,1 & 0,18 & 175 & $6,40 E-05$ & $3,30 \mathrm{E}-03$ & $8,18 \mathrm{E}-04$ \\
\hline
\end{tabular}

Pour cela, nous calculerons tout d'abord le coefficient de corrélation linéaire existant entre les perméabilités estimées et les perméabilités mesurées. Celui-ci vaut :

$$
r=\frac{\operatorname{Cov}(k p, k e)}{\text { Skp Ske }}
$$

avec:

kp perméabilité par pompage :

ke perméabilité estimée

$\mathrm{mx}$ moyenne de la variable $\mathrm{x}$;

Sx écart-type de la variable $x$.

$$
\operatorname{Cov}(x, y)=\frac{1}{n} \sum_{i=1}^{n}(x i-m x)(y i-m y)
$$

Les valeurs des coefficients de corrélation linéaires obtenus sont les suivantes:

\begin{tabular}{l|c|c}
\hline & $\begin{array}{c}\text { Corrélation } \\
\text { k pompage/k Hazen }\end{array}$ & $\begin{array}{c}\text { Corrélation } \\
\text { k pompage/ }\end{array}$ \\
\hline Valeur de $\mathrm{r}$ & 0,768 & 0,83 \\
\hline
\end{tabular}

La corrélation de $k$ mesuré par pompage est donc meilleure avec $k_{c}$ estimé par la formule (9) qu'avec $k$ Hazen estimé en utilisant la formule de Hazen lorsque $\frac{\mathrm{d}_{0,0}}{\mathrm{~d}_{10}}>2$.

Nous avons étudié par ailleurs la distribution des variables :

$C_{H}=\log (k$ Hazen $)-\log (k$ pompage $)$

$C_{\mathrm{i}}=\log \left(k_{\mathrm{G}}\right)-\log (k$ pompage).

Nous donnons, figures 1 et 2, les histogrammes et les ajustements à des lois normales de ces deux variables.

On constate que $\mathrm{C}_{H}$ s'ajuste sur une distribution normale de paramètres :

$\begin{array}{ll}\text { - moyenne } & \mu=-0,99 ; \\ \text { - écart-type } & \sigma=0,96 .\end{array}$

Pour l'échantillon testé, on a, donc, en moyenne: $\ln \left(\frac{\mathrm{k} \text { Hazen }}{\mathrm{k} \text { pompage }}\right)=-0,99$ soit $\mathrm{kHazen}=0,37 \mathrm{k}$ pompage

L'estimation par la formule de Hazen conduit donc, pour l'échantillon testé, à sous-estimer en moyenne la perméabilité d'environ un facteur 3 . 


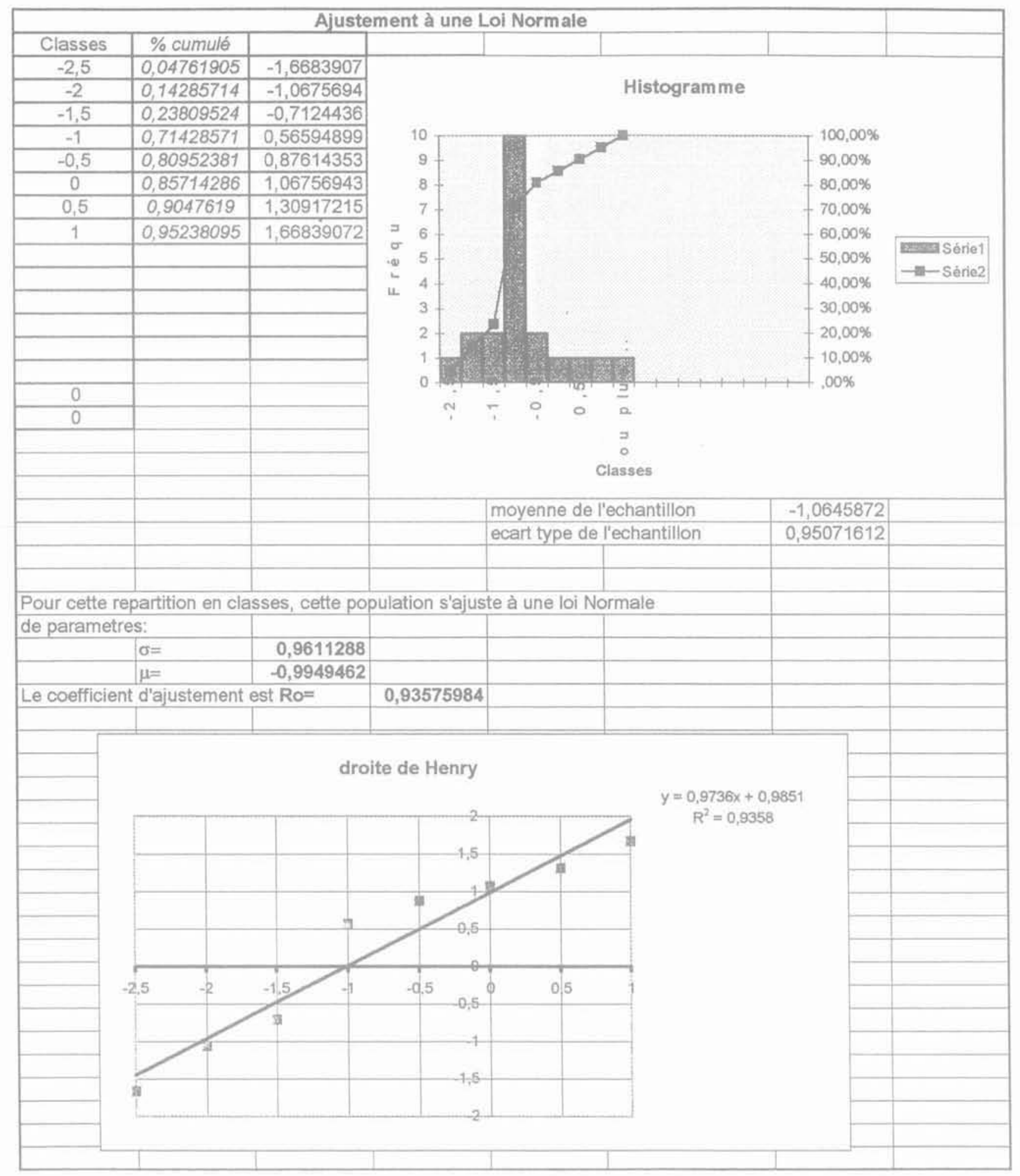

FG, I Ajustement à une loi normale de la variable $C_{H 1}=\log (k$ Hazen $/ \mathrm{k}$ pompage).

On constate, par contre, que $\mathrm{C}_{\mathrm{G}}$ s'ajuste très bien sur une distribution normale de paramètres:

$$
\begin{array}{ll}
\text { - moyenne } & \mu=0,014 ; \\
\text { - écart-type } & \sigma=0,775 .
\end{array}
$$

Pour l'échantillon testé, on a, donc, en moyenne : $\log \left(\frac{k_{G}}{k \text { pompage }}\right)=0,014$ soit $k_{G}=1,014 \mathrm{k}$ pompage

Pour l'échantillon testé, l'estimation de la perméabilité par la formule (9) est donc en moyenne identique à la mesure par pompage. 


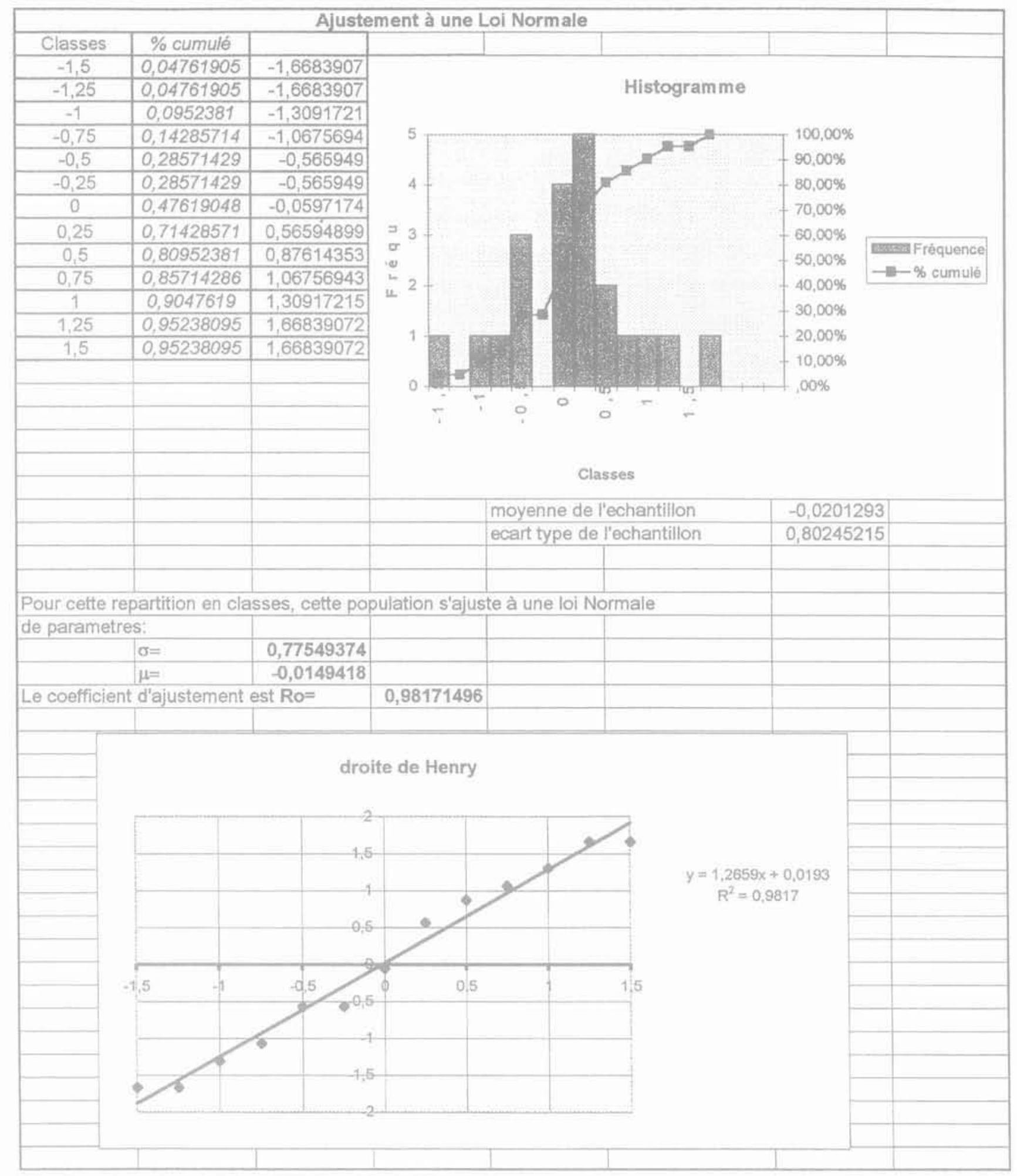

FiG. 2. Ajustement à une loi normale de la variable $\mathrm{C}_{\mathrm{G}}=\mathrm{Log}\left(\mathrm{k}_{\mathrm{G}} / \mathrm{k}\right.$ pompage $)$.

L'intervalle de confiance à $80 \%$ de $\mathrm{C}_{\sigma}$ est:

$\mathrm{I}=0,014 \pm \mathrm{U}_{0,90} \times 0,775=0,014 \pm 1,29 \times 0,775$ soit: $I=0,014 \pm 1$

avec :

$\mathrm{U}_{\text {giso }}$ valeur de la variable aléatoire centrée réduite pour une probabilité de 0,9.
Ceci peut s'écrire : $\frac{1}{10}<\frac{k_{G}}{k \text { pompage }}<10$

En utilisant la formule (9), la probabilité d'avoir une estimation de la perméabilité ne différant pas de plus d'un facteur 10 de la perméabilité vraie est donc de $80 \%$, 


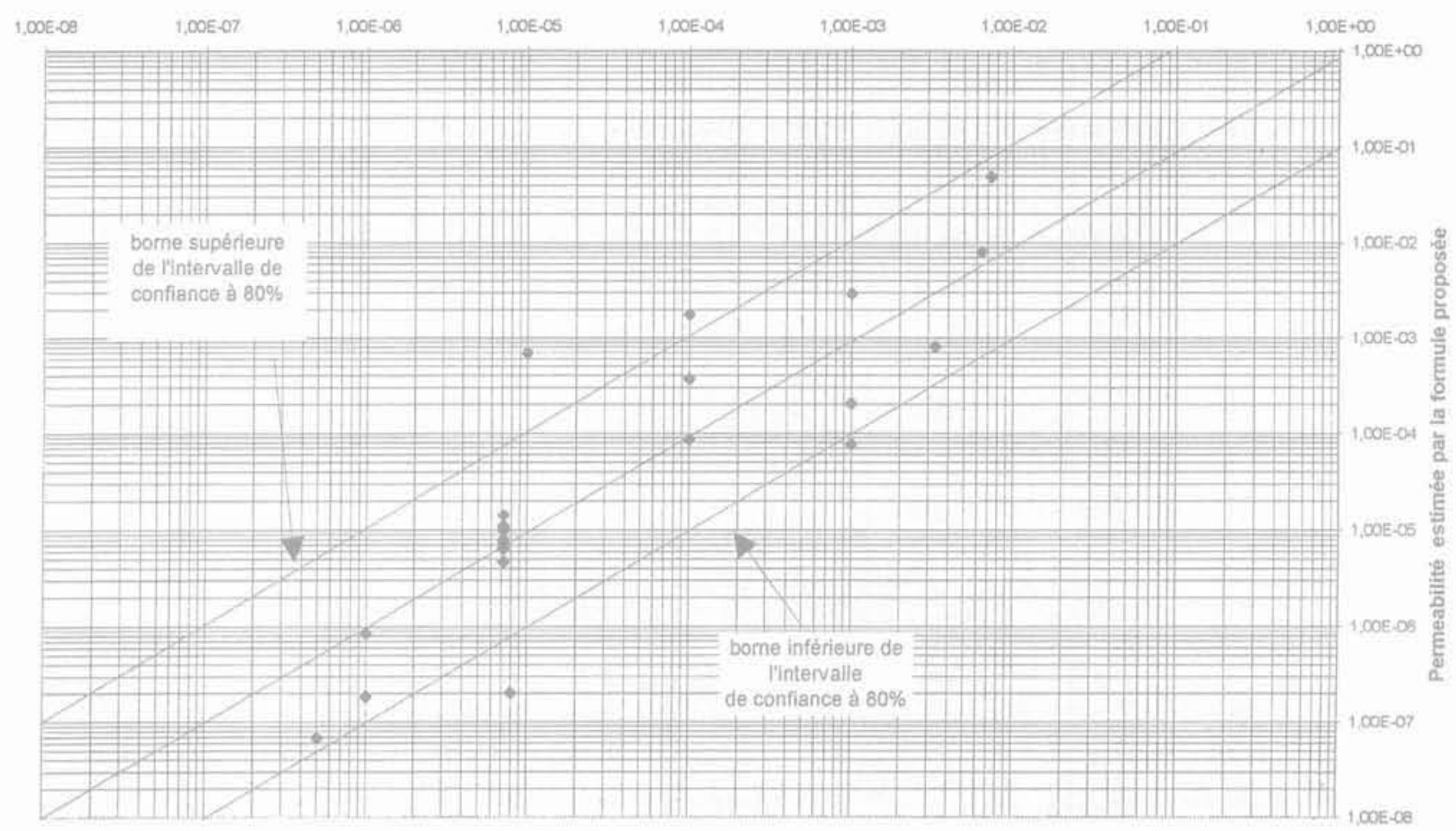

fig. 3 Représentation graphique de l'échantillon testé.

\section{Conclusion}

La formule proposée permet donc pour les sols sablo-limoneux de qranulométrie étalée testés une estimation de meilleure qualitè que la formule de Hazen employée hors de son domaine d'application.

La dispersion n'est pas négligeable et doit conduire à ne pas employer seule cette nouvelle formule.
On pourra, par contre, l'utiliser avec profit pour tester la fiabilité d'essais Lefranc par injection dans les sols sablo-limoneux.

Rappelons que la formule proposée est établie pour des grains sphériques. Elle ne pourra être utilisée pour des sols s'éloignant trop de cette hypothèse et en particulier pour les sols comportant plus de $30 \%$ d'argile au sens minéralogicue du terme.

\section{Bibliographie}

[1] Schneebeli G. - Hydrauligue souterraine, Paris; Eyrolles, 1987 , p. 56-57.

[2] Guéquen Y.. Palciauskas. V. - Introduc. tion à la physigue des roches, Herman Éditeur, 1992, p. 129-130.
[3] Cassan M. Aide mémoire dhydraulique souterraine, Paris, Presses des Ponts et Chaussées, 1993, p. 13

[4] Philiponnat G. - Fondations et ouvrages en terre, Paris, Eyrolles, p. 28-29
[5] Filiat-La pratique des sols et fondation. Éditions du Moniteur. p. 12002-1313. 